\title{
Lineamientos en soporte nutricional en posoperatorios de cirugía cardíaca neonatal
}

\author{
Introduction on postoperative nutritional support in neonatal \\ cardiac surgery
}

\author{
Dra. Vanesa Verónica Oeschger ${ }^{a}$ Dra. Carmen Silvia Mazza \\ Dra. María Beatriz Araujo y Dra. Carola Sauréa
}

\section{RESUMEN}

La malnutrición es una complicación habitual de los neonatos con patología cardiovascular en el período posquirúrgico. Esto sucede por la escasa reserva metabólica, el aumento del gasto energético provocado por la lesión, el aporte de nutrientes insuficiente o tardío que reciben y por su incapacidad de metabolizarlos dada su condición crítica.

Una intervención nutricional adecuada, que alcanza los objetivos, logra mejor respuesta metabólica del neonato operado y tiene consecuencias significativas en el tiempo de internación, cicatrización de heridas, susceptibilidad a las infecciones y evolución posquirúrgica.

Estos lineamientos pretenden establecer fundamentos prácticos para el soportenutricional tanto enteral como parenteral del paciente cardiovascular neonatal, teniendo en cuenta la restricción hídrica y la optimización de macro y micronutrientes requeridos en el posoperatorio. Palabras clave:cardiopatía, cirugía cardiovascular, soporte nutricional, nutrición enteral, nutrición parenteral.

\begin{abstract}
Malnutritionis commoninnewborn patients after cardiac surgery, because of the low metabolic reserves, increased energy expenditure caused by the injury, and reduced or delayed nutritional support they receive, as well as their inability to metabolize the nutrients administered.

It is important to achieve appropriate nutrition; a better metabolic response after surgery has a significant impact on length of stay, wound healing, susceptibility to infections and surgical outcome.

This guideline intended to establish the practical foundation for parenteral and enteral nutritional support in the newborn with cardiac surgery, considering water restriction, optimizing macro and micronutrients required in the postoperative time.

Key words: cardiac anomalies, cardiovascular surgical procedure, nutritional support, enteral nutrition, parenteral nutrition.
\end{abstract}

a. Servicio de Nutrición y Diabetes, Hospital Nacional de Pediatría "Prof. Dr. Juan P.

Garrahan". Buenos

Aires, Argentina.

Correspondencia:

Dra. Carola Sauré: gocarola@fibertel. com.ar

Conflicto de intereses: Ninguno que declarar.

Recibido: 4-2-2014 Aceptado: 12-2-2014 http:/ / dx.doi.org/10.5546/aap.2014.443

\section{INTRODUCCIÓN}

La falla de crecimiento es un problema habitual en los neonatos sometidos a cirugía cardiovascular, que se presenta en el período posquirúrgico inmediato, de etiología multifactorial; su reconocimiento es importante porque el impacto puede ser disminuido a través de intervenciones nutricionales precoces. ${ }^{1-5}$

Como mecanismos, se reconocen el aumento del gasto energético provocado por la lesión; la escasa reserva metabólica de los pacientes pediátricos $\mathrm{y}$, en especial, de los neonatos; la disminución del ingreso neto de nutrientes (por bajo aporte o por su incapacidad de metabolizarlos dada su condición crítica) y el retraso en la implementación del apoyo nutricional. ${ }^{4,5}$

Las intervenciones nutricionales tempranas, cuando se alcanzan los objetivos en el aporte, producen cambios en la respuesta metabólica, con resultados significativos en menor tiempo de internación, mejor evolución de la cicatrización de heridas, menor susceptibilidad a las infecciones y mejor respuesta en la evolución posquirúrgica. ${ }^{4,5} \mathrm{El}$ objetivo de esta pauta es establecer fundamentos básicos para el soporte nutricional enteral y parenteral en el recién nacido con cirugía cardiovascular para optimizar el aporte de macro y micronutrientes para cubrir los requerimientos, $y$, de este modo, mejorar respuestas a corto y mediano plazo.

\section{IMPORTANCIA DEL SOPORTE NUTRICIONAL}

La incidencia de cardiopatías congénitas es del 5 al 8 por mil nacidos 
vivos, por lo que en nuestro país nacen anualmente alrededor de 5000 niños con la patología. El 70\% de estas cardiopatías (aproximadamente, 3500 niños) requiere corrección quirúrgica durante el primer año de vida. ${ }^{6}$

Producto del avance en el diagnóstico y el tratamiento, alcanza la edad adulta un número cada vez mayor de pacientes con cardiopatías complejas. El soporte nutricional constituye uno de los mayores desafíos en la evolución de estos pacientes; el objetivo es lograr una mejor evolución, con disminución de la mortalidad, y optimizar la situación nutricional pre y posquirúrgica que habitualmente se encuentra comprometida.

\section{CAMBIOS METABÓLICOS DEL NEONATO POSQUIRÚRGICO CARDIOVASCULAR}

Luego de una cirugía que requiere bypass cardiopulmonar, los neonatos experimentan una profunda respuesta metabólica al estrés. Esta presenta características diferentes de la de los niños mayores y adultos debido a que los recién nacidos tienen menor reserva metabólica, que los hace extremadamente vulnerables al impacto negativo inducido por el estrés, lo que condiciona una menor cicatrización de heridas y la posterior falla en el crecimiento.

Los cambios metabólicos asociados al estrés incluyen un incremento transitorio en el gasto metabólico basal, que retorna a los valores basales luego de 12-24 h. El aumento es mayor cuando la cirugía ocurre dentro de las primeras $48 \mathrm{~h}$ de vida, que es la situación mas frecuente. ${ }^{3}$

La respuesta metabólica al estrés es mediada por la secreción de citoquinas y mediadores inflamatorios y por la depleción de la ya escasa reserva de glucógeno, que van seguidas de movilización de aminoácidos desde el músculo esquelético como sustrato para gluconeogénesis, glucosa imprescindible para ser utilizada como energía para sostén de la función de órganos vitales y reparación de tejidos. Este proceso, que es un $25 \%$ mayor en el neonato que en los niños mayores, es crucial en los recién nacidos. ${ }^{3,4}$

La respuesta inflamatoria también es exagerada en los neonatos sometidos a cirugía cardiovascular y se acompaña frecuentemente de edema generalizado, capilaritis y falla multiorgánica. Afecta, además, la síntesis de proteínas transportadoras y aumenta la síntesis de reactantes de fase aguda, como la proteína C reactiva $(\mathrm{PCR})$, ceruloplasmina, fibrinógeno y haptoglobina. ${ }^{3}$

El dolor es otro de los factores desencadenantes en la respuesta inflamatoria, que repercute en el estado nutricional. ${ }^{3}$

Si toda esta respuesta ocurre sin soporte nutricional adecuado, la malnutrición lleva a la pérdida de masa magra y al deterioro de la función de órganos vitales, compromiso respiratorio y riesgo de arritmias.

\section{FUNDAMENTOS DEL SOPORTE NUTRICIONAL}

Como ya se mencionó, el período posoperatorio a la cirugía cardíaca es complejo y multifacético y la nutrición juega un rol fundamental dentro de los cuidados.

Así como el aporte insuficiente se asocia con deterioro de la función inmune, dificultades en la cicatrización de heridas y dificultades para el destete del soporte ventilatorio, también la sobrealimentación está asociada con un incremento en la producción de dióxido de carbono, disfunción del sistema inmune y deterioro funcional de los órganos..$^{7-10}$

Aunque el crecimiento es crucial para los neonatos, no es una prioridad durante el posoperatorio inmediato.

Son objetivos del soporte nutricional:

- Inicialmente, preservar la función de órganos vitales, minimizando la pérdida de masa magra, aunque no se pueda evitar completamente el catabolismo.

- Lograr balance nitrogenado positivo, crucial para lograr anabolismo.

- Evitar la sobrealimentación, que condiciona la retención de dióxido de carbono $\left(\mathrm{CO}_{2}\right)$, dificultad en el destete del respirador y alteración de la función inmunológica.

- Cuando hay complicaciones asociadas al posoperatorio (falla renal aguda o quilotórax), los objetivos están supeditados a las indicaciones nutricionales que son específicas para el tratamiento de estas condiciones. ${ }^{3}$

\subsection{Requerimientos: agua, calorías, proteínas, hidratos de carbono, lípidos}

Cada paciente (recién nacido a término, pretérmino, lactante) tiene necesidades nutricionales específicas para mantener un crecimiento y desarrollo adecuados, que deben considerarse al implementar el soporte para evitar la malnutrición.

Se sugiere utilizar las tablas recomendadas para lactantes alimentados con fórmulas comerciales 
y lactancia materna según FAO (Food Agricultural Organization)/OMS (Organización Mundial de la Salud ${ }^{11}$ (Tabla 1) y recomendaciones de European Society of Paediatric Gastroenterology, Hepatology and Nutrition (ESPHGAN) para pacientes con nutrición parenteral ${ }^{12}$ (Tabla 2).

\subsection{Soporte nutricional parenteral \\ Solución estándar}

Siguiendo las recomendaciones para edad y estado crítico, se diseñó una solución estándar de inicio para cubrir requerimientos durante la recuperación inicial considerando la restricción hídrica. ${ }^{3,8,10,12,13}$

\section{Densidad energética}

Los estudios mediante calorimetría indirecta estiman que el consumo calórico del niño crítico varía entre 40 y $65 \mathrm{kcal} / 100$ calorías metabolizadas por día, por lo que es el aporte que se recomienda al iniciar el soporte nutricional., ${ }^{3,4,15}$

Basado en la respuesta metabólica al estrés que experimenta el neonato crítico luego de una cirugía cardiovascular, se sugiere un aporte energético basal de $55-60 \mathrm{kcal} / \mathrm{kg} /$ día, teniendo como objetivo final llegar a $90-100 \mathrm{kcal} / \mathrm{kg} /$ día. $^{3}$

Se estima que se alcanza un balance adecuado que cubre requerimientos calóricos con una solución que tenga $1 \mathrm{kcal} / \mathrm{ml}$, que puede alcanzar

TABLA 1. Requerimientos nutricionales para lactantes alimentados con lactancia materna y fórmulas comerciales de 0 a 3 meses*

Energía: 95-110 kcal/kg/día.

Proteínas: 1,36-1,77 g/kg/día (FAO 2002).

Hidratos de carbono: $50 \%$ de calorías aportadas.

Lípidos: 31 g/día.

* Report of a Joint FAO/WHO/ONU Expert Consultation. Human Energy Requirements. Food and Nutrition Technical Report Series. FAO, 2002-2004.
$1,5 \mathrm{kcal} / \mathrm{ml}$ en situaciones en las que los pacientes precisan restricción hídrica estricta.

Si la cantidad de volumen necesario para cubrir en forma adecuada las proteínas y calorías con la relación de $1 \mathrm{ml}=1 \mathrm{kcal}$ indicada es mal tolerada, es recomendable intensificar el tratamiento diurético en lugar de continuar disminuyendo el aporte hídrico.

\section{Composición de la solución}

Hidratos de carbono: La dextrosa constituye la mayor fuente de calorías no proteicas y determinante de la osmolaridad en las soluciones parenterales. Un flujo inicial de $7 \mathrm{mg} / \mathrm{kg} / \mathrm{min}$ de glucosa aporta los requerimientos necesarios para neonatos, con progresión de $2 \mathrm{mg} / \mathrm{kg} /$ min día. $^{5}$ El aporte máximo recomendado es de $13 \mathrm{mg} /$ $\mathrm{kg} / \mathrm{min}$ para estos pacientes, que evita efectos indeseables, como lipogénesis y depósito hepático posterior, que se produce cuando se infunden flujos mayores. ${ }^{3,9,12,16,17}$

Se debe evaluar la tolerancia a la glucosa con la determinación de glucosurias seriadas y alertar la presencia de glucosuria mayor de $250 \mathrm{mg} / \mathrm{dl}$ en dos micciones consecutivas. ${ }^{17}$

Proteínas: El aporte proteico es de $3 \mathrm{~g} / \mathrm{kg} /$ día, con el que se previene el balance nitrogenado negativo característico de pacientes críticos neonatales, que permite la síntesis de tejido necesaria para el crecimiento. ${ }^{3,10,12,16,17}$ Este aporte, a su vez, cubre las recomendaciones de American Society for Parenteral and Enteral Nutrition (ASPEN) para pacientes con membrana extracorpórea de oxigenación (ECMO) de frecuente utilización. ${ }^{18}$

Lípidos: La emulsión lipídica es utilizada en la nutrición parenteral como fuente energética de bajo volumen y baja osmolaridad. Su utilización es imprescindible para el aporte de ácidos grasos esenciales. Otro beneficio importante del uso de lípidos en la fórmula es que disminuyen la producción de $\mathrm{CO}_{2}$ comparados con las fórmulas de alto contenido de carbohidratos. ${ }^{10,16,17}$

TABLA 2. Requerimientos en nutrición parenteral según la edad*

\begin{tabular}{|c|c|c|c|c|}
\hline & \multicolumn{2}{|c|}{ Recomendaciones } & \multicolumn{2}{|c|}{ Aporte máximo } \\
\hline & Pretérmino & Término & Pretérmino & Término \\
\hline Líquidos ml/kg/día & $140-160$ & $140-160$ & & \\
\hline Energía (kcal/kg/día) & $110-120$ & $90-100$ & & \\
\hline Aminoácidos (g/kg/día) & $2,5-3,5$ & $2,3-2,7$ & & \\
\hline Hidratos de carbono (g/kg/día) & $6-12$ & $7-10$ & & \\
\hline Lípidos (g/kg/día) & \multicolumn{2}{|c|}{$3-4$} & & \\
\hline
\end{tabular}

* Guidelines on Paediatric Parenteral Nutrition. ESPGHAN. 
Con una solución parenteral con $3 \mathrm{~g} / \mathrm{kg} /$ día de lípidos, que es lo recomendado para la edad, se aporta el $40 \%$ de las calorías no proteicas. ${ }^{3,12,17}$

En caso de trombocitopenia grave inexplicable e hipertrigliceridemia mayor a 250 $\mathrm{mg} / \mathrm{dl}$, se recomienda disminuir la infusión de lípidos y reevaluar, para evitar suspender la infusión lipídica y prevenir la deficiencia de ácidos grasos esenciales. ${ }^{12}$

Electrolitos: El requerimiento de sodio en el posoperatorio neonatal es $2-5 \mathrm{mEq} / \mathrm{kg} /$ día; su déficit es un importante limitante del crecimiento..$^{3,12,16}$ Teniendo en cuenta que la omisión de sodio en la fórmula parenteral impide agregar fósforo debido a que se aporta en forma de fosfato sódico, se recomienda iniciar con un mínimo de $2 \mathrm{mEq} / \mathrm{kg} /$ día.

El aporte de potasio no se estandarizó en la fórmula debido a la variabilidad clínica de los pacientes, y se dejó su indicación a cargo del equipo tratante de acuerdo con el cuadro clínico.

Calcio/fósforo: La hipocalcemia inhibe la contractilidad miocárdica, mientras que concentraciones adecuadas de fósforo son necesarias para la generación de ATP y, en consecuencia, una función cardíaca óptima. ${ }^{19}$

La restricción de volumen requerida en el posoperatorio inmediato impide un aporte adecuado de calcio y fósforo en la fórmula parenteral, que cubre solo el $60 \%$ del requerimiento para edad.

Aportando los lípidos por separado de los hidratos de carbonos y proteínas (fórmulas 2 en 1), resulta en un mayor aporte de calcio y fósforo debido a la estabilidad de la preparación, con un aporte de hasta $60 \mathrm{mg} / 100 \mathrm{ml}$ de calcio y $30 \mathrm{mg} / 100 \mathrm{ml}$ de fósforo, con una relación Ca /P $2: 1$, según recomendaciones. ${ }^{12}$

Micronutrientes: La fórmula estándar contiene los micronutrientes en concentraciones según requerimientos de guías ESPGHAN. ${ }^{6,12,16}$ Está indicado suplementar con carnitina a $10 \mathrm{mg} / \mathrm{kg}$ / día, y su déficit constituye riesgo en prematuros dada su función para el transporte de ácidos grasos esenciales. ${ }^{5,6}$

\section{NPT Solución estandarizada de inicio para el paciente en posoperatorio cardiovascular}

(Tabla $3 a$ y b).

- Mínimo volumen hídrico de $60 \mathrm{ml} / \mathrm{kg}$ /día.

- Calorías: 55-60 kcal/kg/día (basal); objetivo: 90-100 cal/kg/día.

- Carbohidratos: 40-60\% del total de kcal. Flujo: $7 \mathrm{mg} / \mathrm{kg} / \mathrm{min}$.

- Aminoácidos: $3 \mathrm{~g} / \mathrm{kg} /$ día.

- Lípidos: 3 g/kg/día, máximo de 0,13-0,17 g/ $\mathrm{kg} / \mathrm{hora}$.

- Sodio: $2 \mathrm{mEq} / \mathrm{kg} /$ día.

- Potasio: debe ser indicado según necesidades del paciente.

- Calcio aportado: $60 \mathrm{mg} / 100 \mathrm{ml}$ de NPT 2 en 1 (máximo).

- Fósforo aportado: $30 \mathrm{mg} / 100 \mathrm{ml}$ de NPT 2 en 1 (máximo).

TABLA 3. Nutrición parenteral total (NPT). Solución estandarizada de inicio para el paciente en posoperatorio cardiovascular

\begin{tabular}{lc|lc} 
a. Aportes de NPT por kg/día & & b. Aporte de NPT por litro de solución \\
Aporte energético & $55-60 \mathrm{kcal} / \mathrm{kg} /$ día & Componente & Composición 2:1 \\
Hidratos de carbono & $7 \mathrm{mg} / \mathrm{kg} / \mathrm{min}$ flujo inicial & Volumen & Mínimo de $60 \mathrm{ml} / \mathrm{kg} / \mathrm{día}$ \\
Aminoácidos & $3 \mathrm{~g} / \mathrm{kg} /$ día & Dextrosado & $167 \mathrm{~g} / \mathrm{L}$ \\
Lípidos & $3 \mathrm{~g} / \mathrm{kg} / \mathrm{día}(\mathrm{máximo} \mathrm{de}$ & Aminoácidos & $50 \mathrm{~g} / \mathrm{L}$ \\
& $0,13-0,17 \mathrm{~g} / \mathrm{kg} / \mathrm{hora})$ & Lípidos & $3 \mathrm{~g} / \mathrm{kg} / \mathrm{día}$ \\
Sodio & $2 \mathrm{mEq} / \mathrm{kg} /$ día & Sodio & $2 \mathrm{mEq} / \mathrm{kg}$ \\
Calcio & Máximo tolerable & Potasio & $\mathrm{mEq} / \mathrm{kg}$ \\
Fósforo & Máximo tolerable & Calcio & $600 \mathrm{mg} / \mathrm{L}(\mathrm{máximo} \mathrm{tolerable)}$ \\
Magnesio & $0,4 \mathrm{mEq} / \mathrm{kg} /$ día & Fósforo & $300 \mathrm{mg} / \mathrm{L}(\mathrm{máximo} \mathrm{tolerable)}$ \\
Carnitina & $10 \mathrm{mg} / \mathrm{kg} /$ día & Magnesio & $97 \mathrm{mg} / \mathrm{L}$ \\
Cobre & $20 \mathrm{mcg} / \mathrm{kg} /$ día & Cobre & $0,304 \mathrm{mg} / \mathrm{L}$ \\
Zinc & $400 \mathrm{mcg} / \mathrm{kg} /$ día & Manganeso & $0,22 \mathrm{mg} / \mathrm{L}$ \\
Manganeso & $1 \mathrm{mcg} / \mathrm{kg} /$ día & Selenio & $0,008 \mathrm{mg} / \mathrm{L}$ \\
Selenio & $2 \mathrm{mcg} / \mathrm{kg} /$ día & Cromo & $0,004 \mathrm{mg} / \mathrm{L}$ \\
Cromo & $0,2 \mathrm{mcg} / \mathrm{kg} /$ día & Molibdeno & $0,004 \mathrm{mg} / \mathrm{L}$ \\
Molibdeno & $0,25 \mathrm{mcg} / \mathrm{kg} /$ día & &
\end{tabular}


- Magnesio: 0,4 mEq/kg/día.

- Carnitina: $10 \mathrm{mg} / \mathrm{kg} /$ día.

- Micronutrientes:

- Cobre: $20 \mathrm{mcg} / \mathrm{kg} /$ día.

- Zinc: $400 \mathrm{mcg} / \mathrm{kg} /$ día. $^{6}$

- Manganeso: $1 \mathrm{mcg} / \mathrm{kg} /$ día.

- Selenio: $2 \mathrm{mcg} / \mathrm{kg} /$ día.

- Cromo: 0,2 mcg/kg/día.

- Molibdeno: 0,25 mcg/kg/día.

En el caso de requerir continuar con NPT luego de la primera semana del posquirúrgico, mantener:

- Volumen de 110-150 ml/kg/día.

- Calorías: 90-100 cal/kg/día.

- Carbohidratos: $40-60 \%$ del total de kcal.

- Flujo:

- Pretérminos: 10-12 mg/kg/min (16-18 gr/kg/día).

- Recién nacido a término: 7-9 $\mathrm{mg} / \mathrm{kg} / \mathrm{min}$.

- Aminoácidos:

- Término: 3-3,5 g/kg/día.

- Bajo peso para la edad gestacional: 3-4 g/kg/día.

- Lípidos: 3-4 g/kg/día; máxima infusión de $0,13-0,17 \mathrm{~g} / \mathrm{kg} / \mathrm{h}$.

- Calcio aportado: $60 \mathrm{mg} / 100 \mathrm{ml}$ de NPT 2 en 1 (máximo).

- Fósforo aportado: $30 \mathrm{mg} / 100 \mathrm{ml}$ de NPT 2 en 1 (máximo).

\section{Implementación}

Iniciar NPT lo antes posible. Cuando las condiciones clínicas lo permitan, comenzar aporte enteral, idealmente no suspender NPT hasta alcanzar aporte de $100 \mathrm{kcal} / \mathrm{kg}$ / día por vía enteral $^{3,5}$ (Tabla 4, Figura 1).

La administración debe ser por vía central debido a la elevada osmolaridad de la fórmula por la alta concentración de la glucosa debido a la restricción de los líquidos. 3,12,17

El tipo de catéter (no implantable, semiimplantables, etc.) dependerá del tiempo planificado del soporte parenteral. Más explicación sobre el tema nos parece que excede a la finalidad de estos lineamientos.

La forma de preparación es 2 en 1; eso implica la administración de los lípidos envasados por separado. Ambas bolsas se administran en forma continua, en lo posible, en otra vía diferente que la central para evitar que precipiten.

En situaciones en que se dificulte dicha preparación y se deba utilizar fórmulas con componentes todo en uno ( 3 en 1), recordar que esto requiere disminuir el aporte de calcio y fósforo para asegurar la estabilidad de la preparación parenteral.

En caso de que no se pueda mantener la vía central y no se haya alcanzado el objetivo por vía enteral (< $100 \mathrm{cal} / \mathrm{kg} /$ día), la administración

TABLA 4. Estrategias tempranas de soporte

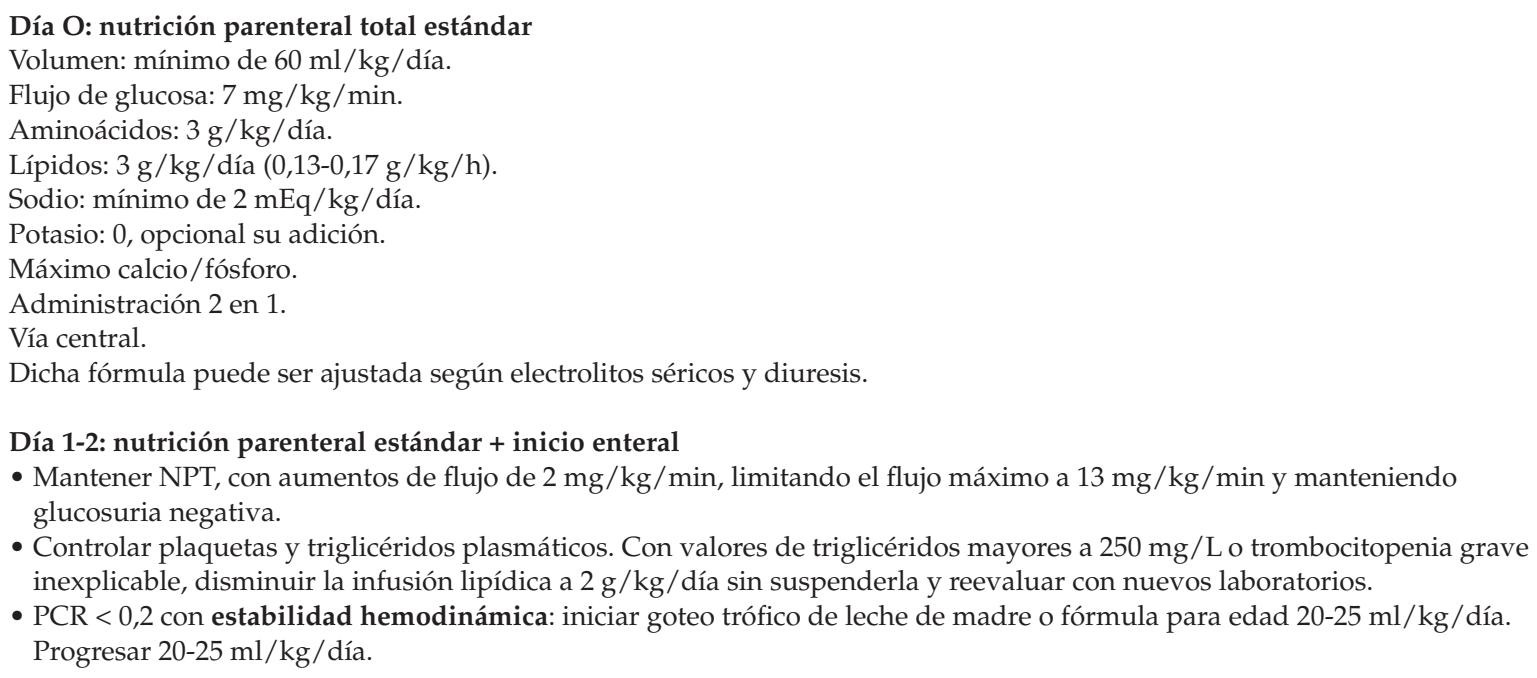

Día 3 en adelante: disminución parenteral + progresión enteral

- Disminuir volumen de NPT hasta suspensión siempre que se haya logrado obtener el 75\% del aporte calórico total en forma enteral.

- Progresar enteral 20-25 ml/kg/día, máximo $150 \mathrm{ml} / \mathrm{kg} /$ día. Una vez obtenido $100 \mathrm{ml} / \mathrm{kg} /$ día, concentrar la fórmula.

- La progresión dependerá del estado clínico y tolerabilidad. 
única de lípidos en forma periférica permite alcanzar la meta calórica. ${ }^{20}$

\section{Monitoreo}

- Control de sodio y potasio plasmático diario.

- Prealbúmina, como marcador de desnutrición proteica semanal. ${ }^{4,10}$

- Ionograma urinario para ajustar la administración de sodio según resultados diariamente.

- Control de PCR a las 24- 48 h.,10
- En situaciones de restricción hídrica, es conveniente monitorizar la osmolaridad urinaria, teniendo en cuenta que un lactante puede concentrar hasta 700-1000 mOsm/L, pero lo deseable es mantener una osmolaridad urinaria entre 300 y $400 \mathrm{mOsm} / \mathrm{L}$.

\section{Limitaciones para alcanzar un adecuado soporte nutricional:}

Son múltiples las situaciones que dificultan el alcance de los objetivos nutricionales:

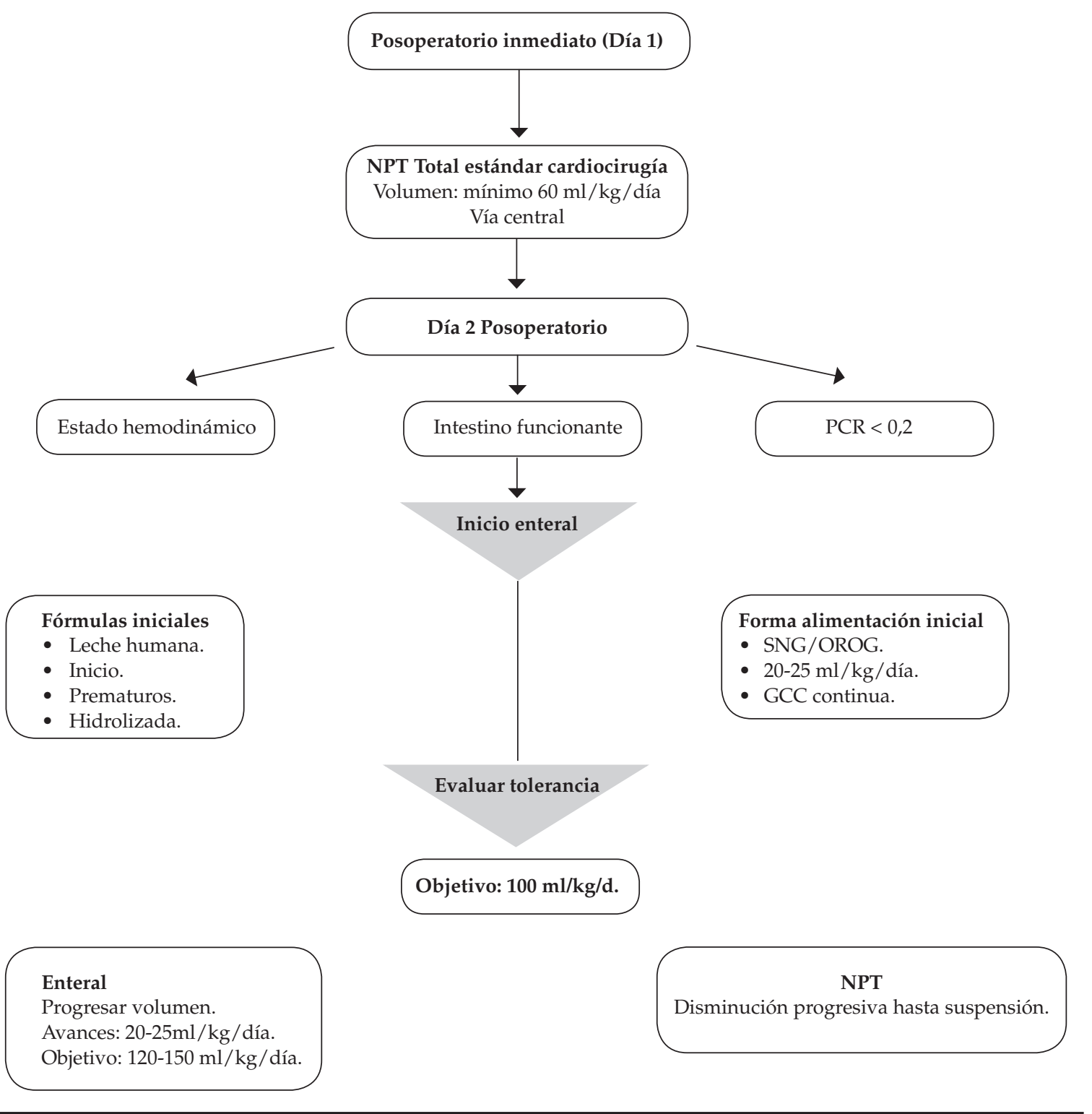

NPT: nutrición parenteral total; SNG: sonda nasogástrica; OROG: sonda orogástrica; GCC: goteo continuo; PCR: proteína $C$ reactiva. 
- Inestabilidad hemodinámica.

- Hipotensión.

- Hiperglucemia.

- Necesidad de restringir el volumen hídrico (esto incluye la medicación, correcciones y otros fluidos), que suele ser entre el 50 y el $80 \%$ de los requerimientos basales.

- Requerimiento de asistencia respiratoria mecánica (ARM).

- Alteración electrolítica y alteración de la función renal (sobre todo, en los pacientes que requieren bypass cardiopulmonar).

- Disminución del output cardíaco posquirúrgico, que genera hipoperfusión (principalmente, en cirugía de Norwood).

- Necesidad de inotrópicos.

\subsection{Soporte nutricional enteral}

El tracto gastrointestinal es la ruta ideal para proveer nutrientes en forma óptima. Cuando no existen contraindicaciones identificables, la alimentación enteral debe iniciarse precozmente. ${ }^{5,10,21}$

Son contraindicaciones para la alimentación enteral:

- Inestabilidad hemodinámica.

- Síntomas de enterocolitis necrotizante (NEC): distención abdominal, sangre en materia fecal y/o débito por sonda nasogástrica. ${ }^{22,23}$
Alimentación enteral para pacientes recién nacidos en posoperatorio de cirugía cardiovascular. Idealmente, se debe realizar con leche materna ${ }^{5,10,23-25}$

Cuando hay limitaciones, se indican fórmulas. Para $\mathrm{RN}$ a término de 0 a 6 meses, es la fórmula de inicio y fórmulas para prematuros en niños pretérminos $^{5,24}$ (Tabla 5). No existe evidencia para la indicación de fórmulas hidrolizadas o sin lactosa en este grupo, por lo que se reserva su utilización en situaciones nutricionales específicas.

Las fórmulas se preparan con las diluciones habituales. Cuando es necesario, pueden ser más concentradas con un incremento de un $5 \%$ máximo, que constituye un método efectivo para incrementar el aporte energético manteniendo la relación de los micro y macronutrientes o aumentar densidad calórica con el agregado de aceite, inicialmente al 1\% y aumentando según tolerancia hasta un máximo de 3\%. ${ }^{26-28}$

Volumen inicial: iniciar con aporte trófico 20$25 \mathrm{ml} / \mathrm{kg}$ /día y progresar según tolerancia., 5,6,27

Progresión: 20-25 ml/kg/día hasta llegar al volumen máximo recomendado de $165-180 \mathrm{ml} /$ $\mathrm{kg} /$ día $^{10,19}$ (Tabla 4). Idealmente, se debe llegar al total del aporte no más allá del séptimo día.

Objetivo: $120-150 \mathrm{ml} / \mathrm{kg} /$ día.

- $1^{\mathrm{er}}$ paso: llegar a volumen máximo tolerable

TABLA 5. Fórmulas enterales de utilización frecuente

\begin{tabular}{|c|c|c|c|c|c|c|c|c|c|c|c|c|c|}
\hline \multirow{2}{*}{$\begin{array}{l}\text { Fórmulas } \\
\text { c/100 ml } \\
\text { Concentración (\%) }\end{array}$} & \multicolumn{2}{|c|}{ Prematuros } & \multicolumn{2}{|c|}{$\begin{array}{c}\text { Inicio } \\
\text { (de } 0 \text { a } 6 \text { meses) } \\
\mathrm{N}^{\circ} 1 \\
\end{array}$} & \multirow[t]{2}{*}{$\begin{array}{c}\text { Inicio + } \\
\text { aceite 3\% + } \\
\text { polímero } 5 \%\end{array}$} & \multicolumn{2}{|c|}{ Kas $1000^{\circledR}$} & \multicolumn{2}{|c|}{ Pepti Junior $^{\circledR}$} & \multicolumn{2}{|c|}{ Neocate ${ }^{\circledR}$} & \multicolumn{2}{|c|}{$\begin{array}{c}\text { Vital } R^{\circledR} \\
\text { (sin lactosa) }\end{array}$} \\
\hline & 16,1 & 21,5 & 13,2 & 17,6 & & 13,5 & 18 & 12,9 & 17,2 & 15 & 20 & 15 & 20 \\
\hline Calorías (kcal) & 80 & 107 & 67 & 89 & $\begin{array}{c}113 \\
(67+27+19)\end{array}$ & 67 & 89 & 66 & 88 & 71 & 94,6 & 73 & 97 \\
\hline $\mathrm{CPRS}^{*}(\mathrm{mOsm} / \mathrm{l})$ & 197 & 262 & 133 & 177 & & 152 & 202 & 108 & 144 & 172 & 229 & 202 & 269 \\
\hline Osmolaridad & 238 & 318 & 259 & 345 & $\begin{array}{c}299 \\
(259+0+40)\end{array}$ & 180 & 240 & 180 & 240 & 360 & 480 & 200 & 267 \\
\hline Proteínas (g) & 2,3 & 3,1 & 1,4 & 1,86 & 1,4 & 2,2 & 2,9 & 1,8 & 2,4 & 1,95 & 2,6 & 2,4 & 3,2 \\
\hline H. de carbono $(\mathrm{g})$ & 8,5 & 11,4 & 7,5 & 10 & 12,5 & 6,9 & 9,2 & 6,8 & 9 & 8,1 & 10,8 & 7,7 & 10,2 \\
\hline Lípidos (g) & 4,6 & 6,2 & 3,5 & 4,6 & 6,5 & 3,4 & 4,5 & 3,5 & 4,6 & 3,5 & 4,6 & 3,6 & 4,8 \\
\hline Sodio (mg) & 46,6 & 62 & 50 & 67 & $\begin{array}{c}28 \\
(25+0+3)\end{array}$ & 54 & 72 & 18 & 24 & 18 & 24 & 42 & 56 \\
\hline Potasio (mg) & 106 & 142 & 72 & 96 & 72 & 82 & 109 & 65 & 86 & 63 & 84 & 97,5 & 130 \\
\hline Calcio (mg) & 136 & 182 & 53 & 71 & 53 & 69 & 92 & 50 & 66 & 49 & 65 & 81 & 108 \\
\hline Fósforo (mg) & 80 & 107 & 33 & 44 & 33 & 47 & 62 & 28 & 37 & 34,5 & 46 & 45 & 60 \\
\hline
\end{tabular}

${ }^{*}$ CPRS: carga potencial renal de solutos. 
progresando 20-25 ml/ kg/ día (de ser necesario, en goteo continuo o intermitente) hasta llegar al volumen deseado., 5,19,27

- $2^{\text {do }}$ paso: cuando no se alcanza la meta calórica con la concentración estándar, aumentar la densidad energética. ${ }^{28}$

Vías: las vías de administración pueden ser a través de sonda nasogástrica, nasoyeyunal, transpilórica o gastrostomía según situación del paciente.

\section{CONCLUSIONES}

El soporte nutricional en el paciente posquirúrgico cardiovascular ha tenido grandes avances en las últimas décadas.

La finalidad de estos lineamientos es establecer una sugerencia de manejo nutricional precoz, en este grupo vulnerable de pacientes, con la primordial finalidad de disminuir los efectos deletéreos del catabolismo, mantener un balance nitrogenado neutro o positivo y proveer los nutrientes recomendados para el mantenimiento normal de las estructuras y funciones.

\section{REFERENCIAS}

1. Cameron JW, Rosenthal A, Olson AD. Malnutrition in hospitalized children with congenital heart disease. Arch Pediatr Adolesc Med 1995;149(10):1098-102.

2. Varan B, Tokel K, Yilmaz G. Malnutrition and growth failure in cyanotic and acyanotic congenital heart disease with and without pulmonay hypertension. Arch Dis Child 1999;81(1):49-52.

3. Owens JL, Musa N. Nutrition support after neonatal cardiac surgery. Nutr Clin Pract 2009;24 (2):242-9.

4. Mehta NM, Duggan CP. Nutritional Deficiencies During Critical Illness. Pediatr Clin North Am 2009;56(5):1143-60.

5. Slicker J, Hehir DA, Horsley M, Monczka J, et al. Nutrition algorithms for infants with hypoplastic left heart syndrome; birth through the first interstage period. Congenit Heart Dis 2013;8(2):89-102.

6. Magliola R, Laura JP, Capelli H. Situación actual de los niños con cardiopatía congénita en Argentina. Arch Argent Pediatr 2000;98(2):130-3.

7. Latifi R. Nutritional therapy in critically ill and injured patients. Surg Clin North Am 2011;91(3):579-93.

8. Herman R, Btaiche I, Teitelbaum DH. Nutrition support in the pediatric surgical patient. Surg Clin North Am 2011;91(3):511-41.

9. Joffe A, Anton N, Lequier L, Vandermeer B, etal. Nutritional support for critically ill children. Cochrane Database Syst Rev 2009;15(2):DC005133.

10. Mehta NM, Compher C, A.S.P.E.N. Board of Directors. A.S.P.E.N Clinical guidelines: nutrition support of the critically ill child. JPEN. J Parenter Entereral Nutr 2009;33(3):260-76.

11. Human Energy Requirements. Report of a Joint FAO/ WHO/ONU Expert Consultation. Rome, 17-24 october 2001. FAO Food and Nutrition Technical Report Series 1. Roma: Food and Agriculture Organization of the United
Nations, 2004. [Acceso: 14 de febrero 2014]. Disponible en: http://www.fao.org/docrep/007/y5686e/y5686e00.htm.

12. Koletzko B, Goulet O, Hunt J, Krohn K, et al. Guidelines on paediatric parenteral nutrition of the European Society of Pediatric Gastroenterology, Hepatology and Nutrition (ESPGHAN) and the European Society for Clinical Nutrition and Metabolism (ESPEN), supported by the European Society of Paediatric Research (ESPR). J Pediatr Gastroenterol Nutr 2005;41(suppl 2):S1-87.

13. HazleMA, Gajarski RJ, YuS, DonohueJ, etal. Fluid overload in infants following congenital heart surgery. Pediatr Crit Care Med 2013;14(1):44-9.

14. Larsen BMK, Goonewardene LA, Field CJ, Joffe AR, et al. Low energy are associated with adverse outcomes in infants after open heart surgery. JPEN J Parenter Enteral Nutr 2013;37(3):254-60.

15. Leong A, Field CJ, Larsen BM. Nutrition support of the postoperative cardiac surgery child. Nutr Clin Pract 2013;28(5):572-9.

16. Gomis Muñoz P, Gómez López L, Martínez Costa C, Moreno Villares JM, etal. Documento de consensoSENPE/ SEGHNP/SEFH sobrenutrición parenteral pediátrica. Nutr Hosp 2007;22(6):710-9.

17. Araujo MB, Escobar N, Mazza C. Nutrición parenteral en el paciente pediátrico. Curso a distancia: Medicina Interna Pediátrica (MIP) Hospital Garrahan 2006;1(3):9-31.

18. Jaksic T, Hull M, Modi PB, Ching YA, et al. A.S.P.E.N. Clinical Guidelines: Nutrition Support of Neonates Supported with Extracorporeal Membrane Oxygenation. JPEN J Parenter Enteral Nutr 2010;34(3):247-53.

19. García Algas F, Rosell Camps A. Nutrición en el lactante con cardiopatía congénita. [Consulta: 13 de febrero de 2014]. Disponible en: http:/ / www.secardioped.org/Descargas/ PyB/LP_cap11.pdf.

20. Schwalbe-Terilli CR, Hartman DH, Nagle ML, Gallagher $\mathrm{PR}$, et al. Enteral feeding and caloric intake in neonates after cardiac surgery. Am J Crit Care 2009;18(1):52-7.

21. Willis L, Thureen P, Kaufman J, Wymore E, et al. Enteral feeding in prostaglandin-dependent neonates: is it a safe practice? J Pediatr 2008;153(6):867-9.

22. del Castillo SL, McCulley ME, Khemani RG, Jeffries HE, et al. Reducing the incidence of necrotizing enterocolitis in neonates with hypoplastic left heart syndrome with the introduction of an enteral feed protocol. Pediatr Crit Care Med 2010;11(3):373-7.

23. Fallon EM, Nehra D, Potemkin AK, Gura KM, et al. A.S.P.E.N. Clinical Guidelines: Nutrition Support of Neonatal Patients at Risk for Necrotizing Enterocolitis. JPEN. J Parenter Enteral Nutr 2012;36(5):506-23.

24. Hay WW Jr. Strategies for feeding the preterm infant. Neonatology 2008;94(4):245-54.

25. Natarajan G, Reddy Anne S, Aggarwal S. Enteral feeding of neonates with congenital heart disease. Neonatology 2010;98(4):330-6.

26. Pillo-Blocka F, Adatia I, Sharieff W, McCrindle BW, et al. Rapid advancement to more concentrated formula in infants after surgery for congenital heart disease reduces duration of hospital stay: a randomized clinical trial. J Pediatr 2004;145 (6):761-6.

27. Braudis NJ, Curley MA, Beaupre K, Thomas KC, et al. Enteral feeding algorithm for infants with hypoplastic left heart syndrome poststage I palliation. Pediatr Crit Care Med 2009;10(4):460-6.

28. Davis A, Baker S. The Use of Modular Nutrients in Pediatrics. JPEN J Parenter Enteral Nutr 1996;20(3):228-36. 